\title{
Somministrazione esogena di kisspeptina per sondare la funzione dei neuroni GnRH-secernenti in pazienti con ipogonadismo ipogonadotropo idiopatico
}

\author{
Fabio Lanfranco $^{1} \cdot$ Stefano Allasia $^{1}$
}

Pubblicato online: 14 marzo 2015

(C) Springer International Publishing AG 2015

\section{Commento a:}

Exogenous kisspeptin administration as a probe of GnRH neuronal function in patients with idiopathic hypogonadotropic hypogonadism.

Y.M. Chan, M.F. Lippincott, J.P. Butler, V.F. Sidhoum, C.X. Li, L. Plummer, S.B. Seminara.

Clin Endocrinol Metab (2014) 99(12):E2762-2771

L'ipogonadismo ipogonadotropo idiopatico (IHH) è una condizione clinica caratterizzata da bassi livelli di steroidi sessuali con gonadotropine plasmatiche ridotte o inappropriatamente normali e alterazioni dello sviluppo puberale. Negli ultimi 20 anni, studi genetici effettuati in pazienti con IHH hanno consentito di identificare diverse mutazioni a carico di geni coinvolti nella migrazione, differenziazione e funzione dei neuroni GnRH-secernenti. Nella pratica clinica, la somministrazione sottocutanea pulsatile di GnRH consente di valutare la funzionalità delle cellule gonadotrope e, quindi, di diagnosticare le forme di IHH con ipofisi integra (es. Sindrome di Kallmann). In maniera analoga, la funzionalità dei neuroni $\mathrm{GnRH}$-secernenti potrebbe essere esplorata tramite somministrazione di kisspeptina, un neuropeptide ipotalamico che sembra avere un ruolo centrale nel regolare l'attività secretoria dei neuroni GnRH-secernenti.

Gli autori di questo studio hanno valutato la funzionalità della rete neuronale GnRH mediante la somministrazione esogena di kisspeptina. Sono stati studiati 11 soggetti con IHH congenito ( 9 maschi e 2 femmine) e un maschio che presentava una forma reversibile di IHH. In tutti i soggetti sono stati eseguiti prelievi ematici ogni 10 minuti per 12-14 ore allo scopo di misurare la secrezione spontanea di $\mathrm{LH}$ e quella indotta da boli endovenosi di kisspeptina $(0,24 \mathrm{nmol} / \mathrm{kg})$ e di GnRH $(75 \mathrm{ng} / \mathrm{kg})$ prima e dopo 6 giorni di terapia con GnRH in somministrazione sottocutanea pulsatile alla dose di $25 \mathrm{ng} / \mathrm{kg}$ ogni 120 minuti. Nessuno degli 11 soggetti con IHH permanente ha esibito una secrezione basale di LH-indipendentemente dal tipo di mutazione genetica identificata $-\mathrm{O}$ una risposta di LH a kisspeptina, sia prima sia dopo trattamento con $\mathrm{GnRH}$. Al contrario, il soggetto con IHH reversibile ha presentato una spiccata risposta a kisspeptina.

Dallo studio è emerso, dunque, che la capacità funzionale della rete neuronale $\mathrm{GnRH}$-secernente risulta essere compromessa nei pazienti con IHH permanente ma non nel soggetto con IHH reversibile, come evidenziato dalla normale risposta a kisspeptina. Ulteriori ricerche sono necessarie per stabilire se l'ipogonadismo ipogonadotropo reversibile, causa di ritardo puberale semplice, espressione clinica di un avvio estremamente procrastinato ma fisiologico del timing puberale, sia legato alla capacità dei neuroni GnRHsecernenti di diventare nel tempo sensibili al neuropeptide kisspeptina.

F. Lanfranco

fabio.lanfranco@unito.it

1 Divisione di Endocrinologia, Diabetologia e Metabolismo, Dipartimento di Scienze Mediche, Università di Torino, Torino, Italia 